suggesting that the results are general, and are not specific to the geometry. The team pointed out that the absorption is significantly enhanced only when the gap between the nanowire surfaces is about $20 \%$ of the nanowire radius. Consequently, the arrays have to be carefully designed, rather than depending on random variation during the fabrication process.

\section{SCANNING TUNNELLING MICROSCOPY} Exploiting expansion

Phys. Rev. Lett. 111, 126101 (2013)

Scanning tunnelling microscopy (STM) is a powerful technique for imaging single molecules on surfaces, and when combined with inelastic electron tunnelling spectroscopy it can provide simultaneous topographical and vibrational information. However, it does not permit optical processes to be directly probed and its spectral resolution is limited by thermal broadening. Now, researchers in California, USA, have devised a new technique called infrared scanning tunnelling microscopy that overcomes these limitations. It essentially involves using an STM tip in tunnelling mode to remotely (at a distance of $\sim 1 \mathrm{~mm}$ ) probe the response of a submonolayer of adsorbed molecules to infrared excitation. The wavelength of a tunable infrared laser is scanned, and the STM tip is used to monitor the thermal expansion of the substrate induced by absorption of the laser light. When the laser wavelength is resonant with a vibration of the absorbed molecules, this expansion is enhanced, producing a peak in the spectrum. The technique can probe a wide range of molecular species with a subthermalenergy resolution that is superior to that of STM-based inelastic electron tunnelling spectroscopy. The researchers demonstrated the effectiveness of this technique in differentiating chemical structures by obtaining clearly distinct spectra from two closely related isomers.

OPTICAL RESONATORS

\section{High-purity diamond}

Opt. Lett. 38, 4320-4323 (2013)

Researchers in the USA and the UK have produced a whispering-gallery-mode resonator made from extremely high purity diamond. By minimizing leakage of impurities into the synthesis chamber and using highpurity gases, they produced a diamond containing only about $20 \mathrm{ppb}$ of nitrogen impurities. The resonator made from this diamond had a $Q$-factor of $2.4 \times 10^{7}$, which is two orders of magnitude higher than the previous highest $Q$-factor for a monolithic diamond resonator. The measured $Q$-factor corresponds to an absorption coefficient of $4 \times 10^{-3} \mathrm{~cm}^{-1}$. Previous measurements of the absorption coefficient of diamond have been dominated by scattering losses from surfaces, and thus do not reflect the true absorption of bulk diamond. In contrast, the researchers estimate that surface scattering contributes only about $10 \%$ to their measured absorption coefficient. Further modest improvements to the diamond purity should enable such resonators to be used in devices such as ultrastable optical etalons, cavity-stabilized lasers and optoelectronic radio-frequency oscillators.

\section{METROLOGY}

\section{Terahertz comb}

\section{Appl. Phys. Express 6, 102202 (2013)}

A highly accurate terahertz frequency counter has been developed by scientists in Japan. The counter could become a vital component of a future clock for frequency standards in the terahertz spectral region. It could also be used to perform precise spectroscopy of ultracold molecules. Hiroyuki Ito and co-workers from the National Institute of Information and Communications Technology in Tokyo, Japan, built the counter by creating a terahertz frequency comb using a photoconductive antenna and a femtosecond mode-locked ytterbium fibre laser. To test the precision of the system, the team made two identical systems and then performed a frequency comparison between them. This revealed a fractional frequency uncertainty of $1.4 \times 10^{-17}$ at $0.3 \mathrm{THz}$ and an instability of $4.1 \times 10^{-14} \tau^{-1}$ for an averaging time of $10,000 \mathrm{~s}$.

\section{QUANTUM OPTICS \\ Subwavelength localization \\ Phys. Rev. X 3, 031014 (2013)}

The ability to localize atomic excitations on the subwavelength scale could help reduce the required spacing for qubits in quantum information processing and increase the speed of two-qubit gates. Researchers from the University of Wisconsin at Madison in the USA have now managed this feat with a spatial width of $100 \mathrm{~nm}$ in a sample of ultracold ${ }^{87} \mathrm{Rb}$ atoms in an optical trap. The team used a laser with a standing-wave pattern to drive electromagnetically induced transparency (EIT) in a sample of atoms. They report that the hyperfine excitation could be localized to a region eight times smaller than the wavelength of the EIT beams. The EIT pulses were 100 ns long, indicating a gate speed capability of about $10 \mathrm{MHz}$. The team now plans to attempt atomic localization at the $10 \mathrm{~nm}$ level by using a different excitation line for the EIT and switching to a longer wavelength for the optical trapping beam in order to cool the atoms to below $1 \mu \mathrm{K}$. The ultimate aim is to create single-qubit and two-qubit gates that operate with a subwavelength spatial resolution.

\section{QUANTUM OPTICS}

\section{Spatial memory}

Nature Commun. 4, 3527 (2013)

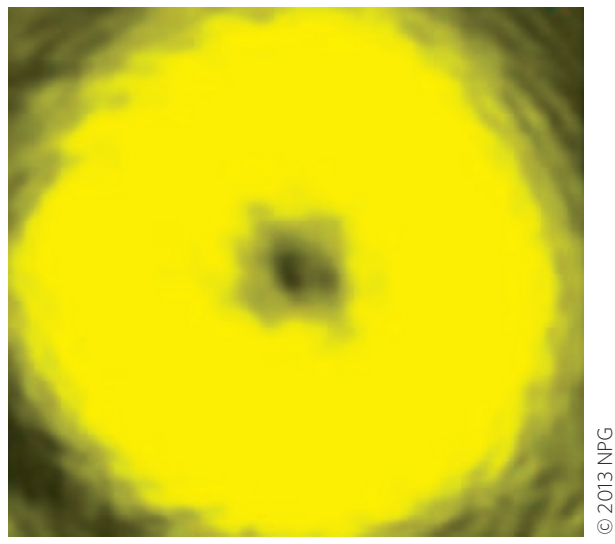

In quantum information science, the ability to transfer a quantum state between a single photon and matter is essential for realizing a quantum memory. Dong-Sheng Ding and co-workers at the University of Science and Technology in Hefei, China, have now demonstrated a quantum memory that operates by using single photons to carry spatial information in the form of orbital angular momentum. The scheme relies on electromagnetically induced transparency in a cold ${ }^{85} \mathrm{Rb}$ atomic ensemble. A proof-of-principle experiment was conducted using non-classical photon pairs generated by spontaneous four-wave mixing in a cold ${ }^{85} \mathrm{Rb}$ atomic ensemble. One photon (wavelength, $780 \mathrm{~nm}$ ) is used as a trigger, whereas the other (wavelength, $795 \mathrm{~nm}$ ) is stored in a second cold ${ }^{85} \mathrm{Rb}$ atomic ensemble. The researchers found that noise mainly originated from a photon generated by a specific atomic transition, and they developed an optical excitation technique to suppress the noise. Photons with an orbital angular momentum state that gave rise to a doughnut-shaped spatial intensity distribution were stored inside the atomic ensemble for $100 \mathrm{~ns}$, and were then retrieved using a single-mode optical fibre. The obtained spatial intensity images of the photons had a similarity of 0.996 , indicating successful operation of the quantum image memory.

Written by Oliver Graydon, Noriaki Horiuchi, David Pile, Simon Pleasants and Rachel Won. 